\title{
Transcriptional regulation of the carbohydrate utilization network in Thermotoga maritima
}

\author{
Dmitry A. Rodionov ${ }^{1,2 *}$, Irina A. Rodionova ${ }^{1}$, Xiaoqing $L^{1}{ }^{1}$, Dmitry A. Ravcheev ${ }^{1,2}$, \\ Yekaterina Tarasova ${ }^{3}$, Vasiliy A. Portnoy ${ }^{3}$, Karsten Zengler ${ }^{3}$ and Andrei L. Osterman ${ }^{1}$ \\ Sanford-Burnham Medical Research Institute, La Jolla, CA, USA \\ ${ }^{2}$ A. A. Kharkevich Institute for Information Transmission Problems, Russian Academy of Sciences, Moscow, Russia \\ ${ }^{3}$ Department of Bioengineering, University of California San Diego, La Jolla, CA, USA
}

Edited by:

Aindrila Mukhopadhyay, Lawrence Berkeley National Laboratory, USA

Reviewed by:

Dong-Woo Lee, Kyungpook National University, South Korea

Aindrila Mukhopadhyay, Lawrence

Berkeley National Laboratory, USA

*Correspondence:

Dmitry A. Rodionov,

Sanford-Burnham Medical Research Institute, 10901 North Torrey Pines

Road, La Jolla, CA 92037, USA

e-mail: rodionov@burnham.org
Hyperthermophilic bacteria from the Thermotogales lineage can produce hydrogen by fermenting a wide range of carbohydrates. Previous experimental studies identified a large fraction of genes committed to carbohydrate degradation and utilization in the model bacterium Thermotoga maritima. Knowledge of these genes enabled comprehensive reconstruction of biochemical pathways comprising the carbohydrate utilization network. However, transcriptional factors (TFs) and regulatory mechanisms driving this network remained largely unknown. Here, we used an integrated approach based on comparative analysis of genomic and transcriptomic data for the reconstruction of the carbohydrate utilization regulatory networks in 11 Thermotogales genomes. We identified DNA-binding motifs and regulons for 19 orthologous TFs in the Thermotogales. The inferred regulatory network in T. maritima contains 181 genes encoding TFs, sugar catabolic enzymes and $\mathrm{ABC}$-family transporters. In contrast to many previously described bacteria, a transcriptional regulation strategy of Thermotoga does not employ global regulatory factors. The reconstructed regulatory network in T. maritima was validated by gene expression profiling on a panel of mono- and disaccharides and by in vitro DNA-binding assays. The observed upregulation of genes involved in catabolism of pectin, trehalose, cellobiose, arabinose, rhamnose, xylose, glucose, galactose, and ribose showed a strong correlation with the UxaR, TreR, BglR, CelR, AraR, RhaR, XylR, GluR, GalR, and RbsR regulons. Ultimately, this study elucidated the transcriptional regulatory network and mechanisms controlling expression of carbohydrate utilization genes in T. maritima. In addition to improving the functional annotations of associated transporters and catabolic enzymes, this research provides novel insights into the evolution of regulatory networks in Thermotogales.

Keywords: carbohydrate metabolism, transcriptional regulation, regulon, comparative genomics, Thermotoga

\section{INTRODUCTION}

Carbohydrates constitute the most abundant single class of organic substances found in nature. Plant cell walls constitute $\sim 70 \%$ of the worldwide biomass production by land plants, but only $\sim 2 \%$ of this biomass is currently utilized by humans (Pauly and Keegstra, 2010). Carbohydrate composition of plant biomass is highly diverse and differs significantly between plants. A limited number of mono-or disaccharides compose a majority of plant biopolymers. Cellulose, hemicelluloses, and pectins are major polysaccharides of the plant cell wall. Hemicelluloses are characterized by a large diversity of building blocks including pentoses (xylose, arabinose) and hexoses (glucose, mannose, galactose, and rhamnose, and uronic acids). Pectins are composed of galacturonate and rhamnose residues with various branching side chains. Microbial degradation of plant cell wall polysaccharides and their conversion to biofuels is a vital objective for society and requires novel efficient technologies.
Sugar utilization pathways are major feed lines of carbon and energy for central metabolism in a large variety of heterotrophic bacteria. Although these pathways and their transcriptional regulation were extensively studied in model bacteria, projection of this knowledge to diverse bacteria is a major challenge due to chemical diversity of carbohydrates and a matching variability of microbial sugar utilization genes, pathways, and regulons. This variability includes alternative biochemical routes, nonorthologous gene replacements, and functionally heterogeneous families of paralogs. Due to this complexity, genomic annotations of sugar utilization genes derived solely from sequence similarity analysis are often imprecise and incomplete, especially for distantly related bacteria. Metabolic reconstruction based on a combination of various types of genomic context analysis (Yang et al., 2006; Rodionov et al., 2007; Leyn et al., 2012), primarily operons and regulons, allows us to substantially improve the quality of functional annotations and predictions, enabling more accurate metabolic modeling. In this study, we applied an integrative 
approach to simultaneous genomics-based reconstruction of both metabolic and associated regulatory networks to study carbohydrate utilization networks in a hyperthermophilic marine bacterium from the phylogenetically deep-branching Thermotogales group.

Thermotoga spp. are anaerobic fermentative bacteria that are able to grow on various simple and complex carbohydrates including glucose, starch, cellobiose, xylan, and pectin while producing hydrogen, carbon dioxide, and acetate (Chhabra et al., 2002; Kluskens et al., 2003; Conners et al., 2006). T. maritima MSB8, a model bacterium in the Thermotoga group, was isolated from geothermally heated marine sediments of Volcano Island in Italy (Huber et al., 1986). A closely related bacterium, T. neapolitana, was isolated from a submarine thermal vent near Lucrino, Bay of Naples, Italy (Jannasch et al., 1988). Other Thermotogales have a broad geographic distribution (see Figure 1) including hydrothermal vents in the Azores [Themotoga sp. RQ-2, (Swithers et al., 2011)], a sulfate-reducing bioreactor in Europe [T. lettingae, (Balk et al., 2002)], a hot spring in New Zealand [Fervidobacterium nodosum, (Patel et al., 1985)], and an offshore oil reservoir in Japan [T. naphthophila, T. petrophila, (Takahata et al., 2000)].
The complete genome sequence of $T$. maritima revealed a large proportion of genes (10-15\%) involved in carbohydrate metabolism (Nelson et al., 1999). A number of T. maritima and T. neapolitana enzymes that can degrade $\beta$-glucan, hemicelluloses, and pectin have been studied by functional genomics or biochemistry (Conners et al., 2006). A three-dimensional reconstruction of the central metabolic network of T. maritima includes 478 enzymes, 120 of which were determined experimentally (Zhang et al., 2009). Still, many metabolic gaps remained in sugar catabolic networks of T. maritima. We recently investigated some of those gaps using a combination of bioinformatics and experimental techniques (Rodionova et al., 2012a,b, 2013).

The comprehensive genome-scale metabolic modeling of $T$. maritima also requires an understanding of mechanisms, components, and behavior of the transcriptional regulatory machinery. The integration of genome-scale metabolic and regulatory models significantly improves growth phenotype predictions and allows for the interpretation of systems biology datasets (Faria et al., 2013). Functional genomics approaches were previously used to study global gene expression in response to growth of $T$. maritima on sugars. The transcriptional responses of $T$. maritima to various mono- and polysaccharides identified subsets

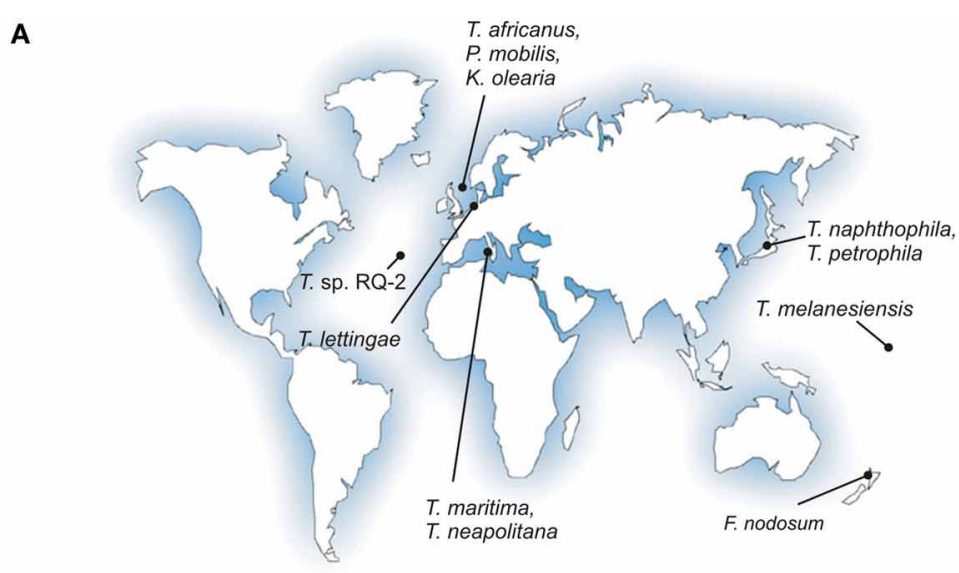

B

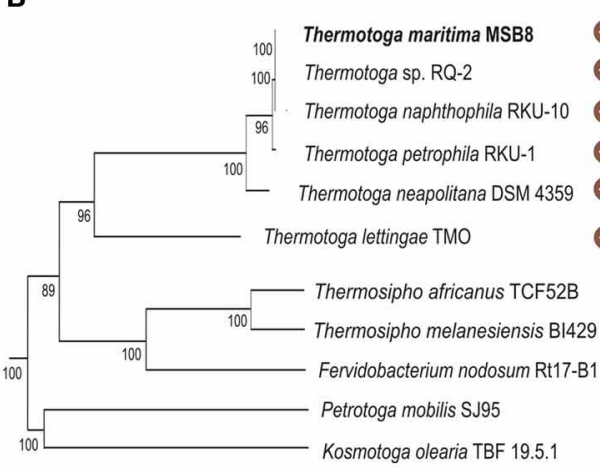

FIGURE 1 |Properties of 11 Thermotogales species analyzed in this study. (A) The geographic isolation sites for the Thermotogales strains. (B) Distribution of sugar utilization pathways and cognate transcriptional regulators. The presence of orthologous genes encoding regulators and associated sugar

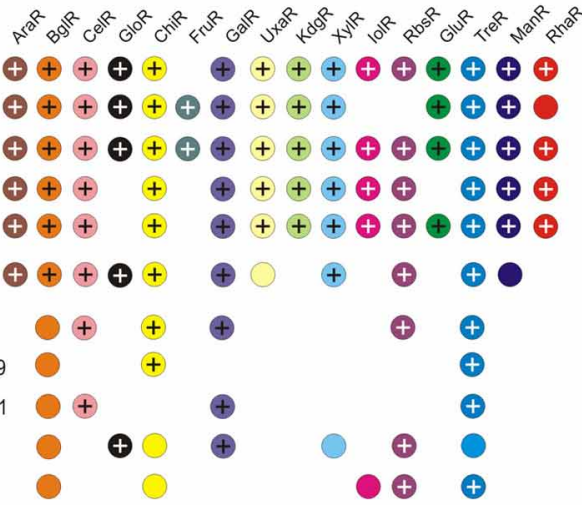

catabolic pathways is shown by "+" and colored circles, respectively. The phylogenetic species tree was constructed using the concatenated alignment of 78 universal bacterial proteins in the MicrobesOnline database (http://www.microbesonline.org/cgi-bin/ speciesTree.cgi). 
of genes that are coordinately regulated in response to specific carbohydrates (Chhabra et al., 2003; Nguyen et al., 2004; Conners et al., 2005; Frock et al., 2012). However, the molecular mechanisms underlying these transcriptional responses remain unclear.

To infer sugar-responsive transcriptional regulons in Thermotoga, we started from the initial metabolic reconstruction, applying a combination of comparative genomics-based methods of regulatory reconstruction (Rodionov, 2007). Previously, we applied these methods to reconstruct a large number of metabolic regulons in diverse taxonomic groups of bacteria (Rodionov et al., 2011; Leyn et al., 2013; Ravcheev et al., 2013). Integration of metabolic and regulatory reconstructions is particularly efficient for elucidation of carbohydrate utilization networks in a group of taxonomically related bacteria, as recently demonstrated by the comparative genomic study of Shewanella spp. (Rodionov et al., 2010). In previous studies, we also combined genomicsbased inferences with experimental elucidation to identify and characterize several novel regulons in Thermotoga, including Rex controlling the central carbon metabolism and hydrogen production (Ravcheev et al., 2012) and seven ROK-family transcription factors controlling the sugar utilization pathways (Kazanov et al., 2013).

Here, we extended this analysis toward the entire transcriptional network for sugar catabolism in T. maritima compared to 10 additional Thermotogales species with completely sequenced genomes. The reconstructed networks allowed us to improve gene annotations, refine associated pathways, and identify novel, yet uncharacterized, enzymes and transporters tentatively implicated in the sugar utilization machinery. Some of the critical inferences about newly identified regulons were experimentally verified. A comparison with global transcriptomic data obtained for T. maritima grown on different carbohydrates provided additional validation and enrichment of the genomic reconstruction.

\section{RESULTS \\ GENOMIC RECONSTRUCTION OF SUGAR CATABOLIC REGULONS IN THERMOTOGALES}

Initially, we used the subsystems-based bioinformatics workflow (see Methods for details) to identify the repertoire of known and putative genes involved in carbohydrate utilization in T. maritima (Table S1 in Supplementary Material). Functional annotation of the identified genes was conducted using the comparative genomic analysis of predicted gene operons and regulons in the Thermotogales that was combined with homology searches and metabolic reconstruction. The identified set of $\sim 240$ genes encodes the components of at least 19 peripheral sugar utilization pathways as well as the central carbohydrate metabolism (Table 1). In T. maritima, the predicted sugar catabolome utilizes 127 enzymes, 22 transport systems (encoded by 86 genes), 18 DNA-binding transcriptional factors, (TFs), and 1 RNA-binding regulator $(\mathrm{GlpP})$.

Next, we identified orthologs for the T. maritima sugar catabolic, transport, and regulatory genes in 10 additional Thermotogales species with complete genomes (Table S2 in Supplementary Material). Orthologs of the predicted sugar regulators are unevenly distributed among the 11 analyzed genomes (Figure 1). We note that the presence of the orthologous cognate TFs is not ubiquitous for some sugar catabolic pathways. The most conserved TFs in the Thermotogales include TreR and GlpR (present in 10 genomes); GalR, GlpP, and UgtR (present in 9 genomes), as well as CelR and ChiR (present in 8 genomes). Three regulators (AraR, BglR, and XylR) were found only in 6 Thermotoga genomes, whereas four regulators (UxaR, KdgR, ManR, and UctR) are conserved in 5 closely-related Thermotoga spp. Finally, GloR, GluR, InoR, and RhaR have orthologs in a small subset of Thermotoga spp.

To infer novel TF regulons, we applied the comparative genomics-based approach implemented in the RegPredict Web server (see Methods). As a result, we revealed DNA motifs and reconstructed regulons for all 18 DNA-binding TFs associated with sugar catabolism in T. maritima. In addition, we inferred a regulon for the predicted fructose regulator FruR, which is present in two Thermotoga species but not in T. maritima. The glycerol regulon operated by the RNA-binding antiterminator protein GlpP was not studied by comparative genomics.

The obtained TF-binding motifs and respective sets of coregulated operons in T. maritima are illustrated in Figure 2. Regulon content for orthologous TFs in other Thermotogales genomes is available in the RegPrecise database (Novichkov et al., 2010a) and in Table S2 in Supplementary Material. Metabolic content of 15 functionally annotated regulons is visualized on the integrative T. maritima sugar catabolic network (Figure 3). Below, we describe the reconstructed transcriptional regulatory network for sugar catabolism in the Thermotogales in more detail.

The xylose-responsive regulon XylR in T. maritima contains seven operons organized into five chromosomal gene clusters that encode xylose catabolic enzymes (XylA, XylB), a xylosespecific $\mathrm{ABC}$ transporter, a set of hydrolytic enzymes specific for xylan and xylan oligosaccharides, and two xylan-induced $\mathrm{ABC}$ transporters. XylR belongs to the ROK (repressor, open reading frame, kinase) protein family. All six predicted XylRbinding sites have been experimentally validated in vitro in our previous study of ROK-family regulators (Kazanov et al., 2013). The XylR regulon is well conserved in five Thermotoga spp., whereas T. lettingae has a reduced size of an orthologous regulon.

The predicted regulator $\mathrm{KdgR}$ in $\mathrm{T}$. maritima (IclR protein family) is homologous to a repressor of the 2-keto-3-deoxygluconate (KDG) metabolism and pectin utilization in enterobacteria (Rodionov et al., 2004). However, in Thermotoga spp., the reconstructed $\mathrm{KdgR}$ regulon does not include the KDG metabolism genes ( $k d g K, k d g A$ ) but is predicted to control the uronate isomerase $u x a C$, encoding a bifunctional enzyme in the catabolism of glucuronate and galacturonate. Additional candidate KdgR-binding sites were identified in the promoter regions of two XylR-regulated gene clusters, $x y n B / x l o$ and $x y n A / x t p$, that are induced during the growth of $T$. maritima on xylan. Glucuronoxylan is a linear polymer of $\beta$-linked xylose residues with many of the xylose units substituted with glucuronate residues. The $x t p$ gene cluster encodes $\alpha$-glucuronidase AguA that can release glucuronate residues from glucuronoxylan. Thus, we propose that the two Thermotoga loci under dual regulation by 
Table 1 | Distribution of the carbohydrate utilization genes and regulators in T. maritima.

\begin{tabular}{|c|c|c|c|c|c|c|}
\hline $\begin{array}{l}\text { Sugar utilization } \\
\text { pathway }\end{array}$ & Abbrev. & Regulators & Transporters & Enzymes & $\begin{array}{l}\text { TOTAL } \\
\text { genes }\end{array}$ & $\begin{array}{l}\text { TOTAL genes in } \\
\text { regulons }\end{array}$ \\
\hline Central carbohydrate metabolism & $\mathrm{CCM}$ & - & - & 18 & 18 & - \\
\hline$\beta$-glucosides & $\mathrm{Bgl}$ & $B g \mid R$ & BglEFGKL, & 2 & 9 & 9 \\
\hline Cellobiose & Cel & CelR & CelEFGKL & 7 & 13 & 13 \\
\hline Xyloglucan oligosaccharides & Glo & GloR & GloEFGKL & 3 & 9 & 9 \\
\hline Fructose & Fru & FruR $^{*}$ & FruAB* & $2^{*}$ & $7^{*}$ & $6^{*}$ \\
\hline Galactose, galactosides & Gal & GalR & LtpEFGKL, GanEFG & 7 & 16 & 15 \\
\hline Glycerol & Glp & GlpP & GIpABC, GlpF & 2 & 7 & - \\
\hline Digalacturonate, pectin & Uxa & UxaR & AguEFG & 15 & 19 & 16 \\
\hline Glucuronate & Kdg & KdgR & - & 1 & 3 & 3 \\
\hline Glucose & Glu & GluR & GluEFK & - & 4 & 4 \\
\hline Trehalose & Tre & TreR & TreEFG & 1 & 5 & 5 \\
\hline Mannose, mannosides & Man & ManR & MtpEFGKL & 7 & 13 & 11 \\
\hline Rhamnose, rhamnose oligosaccharides & Rha & RhaR & RtpEFGKL & 8 & 14 & 14 \\
\hline Arabitol, mannitol & Pol & - & - & 3 & 3 & - \\
\hline Hypothetical sugar utilization & $\mathrm{HSU}$ & UgtR, UctR, UgpR & UgtEFGK，UctMPQ，UgpEFG & 11 & 24 & 20 \\
\hline Total number: & & 19 & 86 & 127 & 241 & 181 \\
\hline
\end{tabular}

The details of all functional assignments summarized in this table are provided in Table S1. Asterisks indicate that FruR regulator and fructose utilization genes are absent from T. maritima and thus were not counted toward the total numbers.

XylR and KdgR are involved in the xylan and glucuronoxylan utilization.

The predicted regulator UxaR (GntR family) was attributed to a conserved 17-nt DNA motif found in multiple copies within the pectin/galacturonate utilization gene loci in five Thermotoga genomes (Rodionova et al., 2012a). The UxaR-regulated pectin catabolic locus in T. maritima includes pectate lyase pelA, digalacturonate $\mathrm{ABC}$ transporter aguEFG, exo-polygalacturonase $p e l B$, and the gnd, $u x a E, u x a D$, gntE, and $g n t K$ genes required for galacturonate catabolism. Interestingly, the T. maritima aguEFG transporter is substituted by other UxaR-regulated transport systems (named uxaPQM and uxaXYZ) in T. petrophila, T. naphtophila, and T. neapolitana. A conserved UxaR binding site was identified upstream of the $k d g A-k d g K-u x u B-u x u A$ operon encoding enzymes of the glucuronate utilization pathway. These results suggest that the pectin/galacturonate and glucuronate catabolic genes are co-regulated by the transcription factor UxaR in Thermotoga spp. The UxaR regulon predicted by in silico genomic analysis was experimentally validated in T. maritima (see Experimental validation of the inferred regulons in T. maritima).

The mannose-responsive regulon ManR (ROK family) includes two operons encoding a set of mannan and mannosides hydrolytic enzymes and a mannan-induced $A B C$ transporter. Both candidate binding sites of ManR and its mannose effector have been previously experimentally tested in T. maritima (Kazanov et al., 2013). The ManR-dependent regulation of the manBDCR operon is conserved in other Thermotoga spp., whereas the ManR-regulated mtp-cel5 operon is conserved only in the RQ-2 strain.

The predicted cellobiose regulator CelR (LacI family) has an extensive regulon including 22 genes from six genomic loci in T. maritima. The conserved part of the CelR regulon (present in five Thermotoga genomes) includes glucan and $\beta$-glucoside degradation enzymes, cellobiose phosphorylase, and a barley glucaninduced $\mathrm{ABC}$ transporter. The latter transporter, termed celE$F G K L$, is also present and regulated by orthologous CelR regulators in three other Thermotogales genomes (Figure 1). In T. maritima, the reconstructed CelR regulon includes an additional $\mathrm{ABC}$ transporter ( $g l o E F G K L)$ encoded within the predicted xyloglucan utilization gene cluster. The LacI-family transcription factor GloR was predicted to function as a local regulator of the glo locus. The common intergenic region of gloR and gloE genes contains distinct operator sites for CelR and GloR regulators. The BglR regulator (ROK family) controls another $\beta$-glucosides utilization operon encoding laminarinase, $\beta$-glucosidase, and cellobioseand laminaribiose-specific $\mathrm{ABC}$ transporters in Thermotoga spp. A BglR-binding site was experimentally validated, and it was found that the BglR regulator from T. maritima responds to two effectors, cellobiose and glucose (Kazanov et al., 2013).

The ROK-family transcription factors GluR and TreR in $T$. maritima control the glucose and trehalose utilization pathways (Kazanov et al., 2013). The trehalose-responsive regulator TreR co-regulates the trehalose $\mathrm{ABC}$ transporter treEFG and the trehalose synthase-like gene treT, whereas GluR controls the glucose $\mathrm{ABC}$ transporter $g l u E F K$ in response to glucose. The 


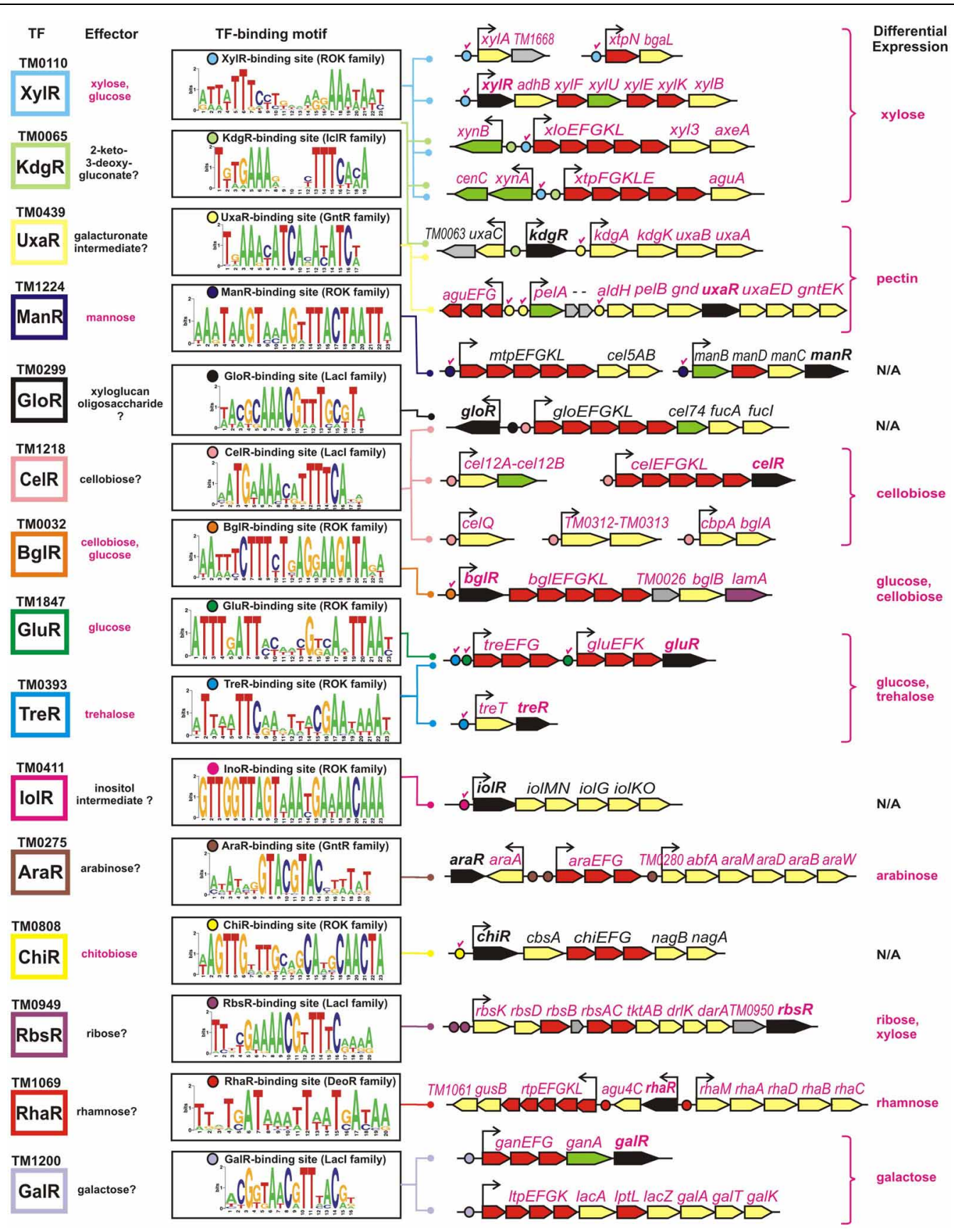

FIGURE 2 | Genomic context of 15 sugar catabolic regulons in $T$. maritima. Validated and predicted effectors of TFs are listed in red and black, respectively. TF binding sites and downstream regulated genes are shown by circles and arrows, respectively. Validated sites are labeled with red check marks. Sequence logos representing the consensus binding site motifs were built using all candidate sites in the Thermotogales genomes.
Genes encoding transcriptional regulators and components of sugar transporters are shown in black and red, respectively, whereas the genes encoding the secreted and intracellular sugar catabolic enzymes are in green and yellow, respectively. Hypothetical genes are in gray. Genes that were upregulated on specific sugars (listed in the last column) have their names highlighted in magenta. glucose regulator has an additional binding site upstream of the treEFG operon, which is conserved in five closely related Thermotoga genomes (Figure 4). These results suggest that the trehalose transporter is under dual control of both regulators. The TreR regulon is conserved in all studied Thermotogales genomes except for Petrotoga mobilis.
Two other ROK-family regulators, IolR and ChiR, control the inositol and chitobiose catabolic operons, respectively. The latter operon includes the predicted chitobiose transporter chiEFG, the chitobiose hydrolase $\operatorname{cbs} A$, and the $\mathrm{N}$-acetylglucosamine (GlcNAc) catabolic genes nagAB. Single DNA binding sites of both IolR and ChiR regulators were experimentally confirmed 


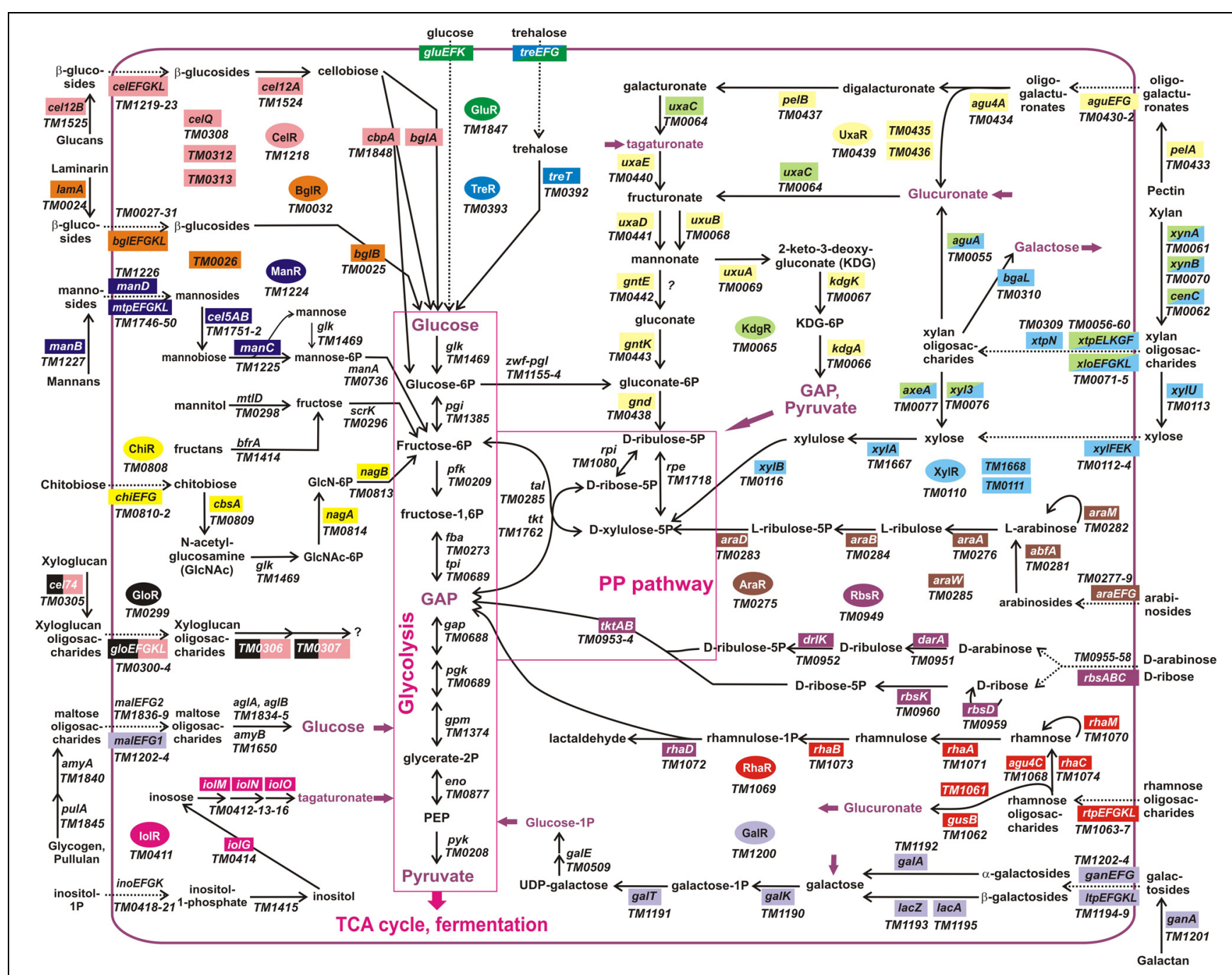

FIGURE 3 | Integrative reconstruction of sugar utilization pathways and regulons in T. maritima. Diagram includes 190 carbohydrate utilization genes encoding catabolic enzymes (solid arrows), transporters (dotted arrows), and transcriptional factors (in ovals of different colors). Genes involved in the reconstructed regulatory network (161 genes regulated by 15 TFs) are highlighted by a matching background color. Three hypothetical sugar utilization pathways and the respective regulons (UgtR, UctR, UgpR) are not shown.
(Kazanov et al., 2013). The specific DNA-binding ability of the ChiR regulator was abolished by a disaccharide chitobiose but not monosaccharide GlcNAc. However, the IolR regulator did not respond to any tested sugar, including glucose, inositol, and inositol phosphate (Kazanov et al., 2013). The IolR regulon is conserved in four Thermotoga genomes, whereas the ChiR regulon is present in all studied Thermotoga and Thermosipho spp. (Figure 1).

The predicted regulator AraR (GntR family) is homologous to the L-arabinose-responsive repressor from Bacillus subtilis (Rodionov et al., 2001). The reconstructed AraR regulon in five Thermotoga species includes predicted $\mathrm{ABC}$ transporters and hydrolytic enzymes for utilization of arabinose oligosaccharides and a set of L-arabinose catabolic enzymes. The predicted ribose regulator RbsR (LacI family) is encoded within the ribose utilization operon. The conserved part of the RbsR regulon in eight Thermotogales genomes includes a ribose-specific
ABC transporter and a ribokinase. In T. maritima, T. neapolitana, and T. lettingae, the ribose operon also includes genes encoding two predicted D-arabinose catabolic enzymes and transketolase.

The predicted rhamnose regulator RhaR (DeoR family) was identified within the rhamnose utilization gene cluster in T. martima and three other Thermotoga spp. The reconstructed RhaR regulon includes a rhamnose-induced $\mathrm{ABC}$ transporter, several glycoside hydrolases, and a set of rhamnose catabolic enzymes. The predicted galactose regulator GalR (LacI family) controls the galactan/galactoside utilization pathways in nine Thermotogales genomes. In T. maritima, the GalR regulon includes two galactose catabolic enzymes, two $\beta$-galactosidases, one galactan hydrolase, and two lactose-inducible ABC transporters. The predicted fructose utilization regulon FruR (DeoR family) was found in only two Thermotoga spp. (T. naphthophila and RQ-2). Finally, three other TF regulons reconstructed in Thermotoga genomes control 


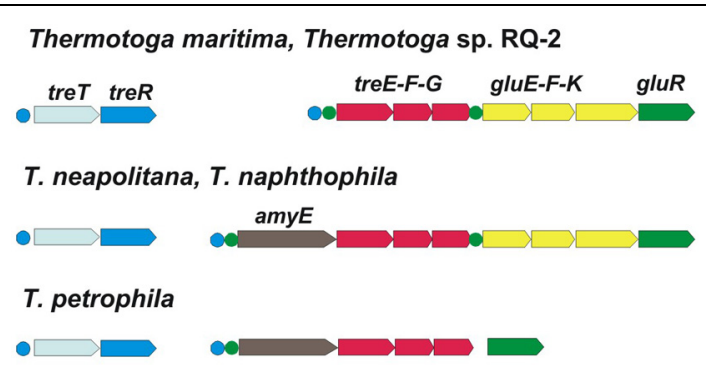

Fervidobacterium nodosum

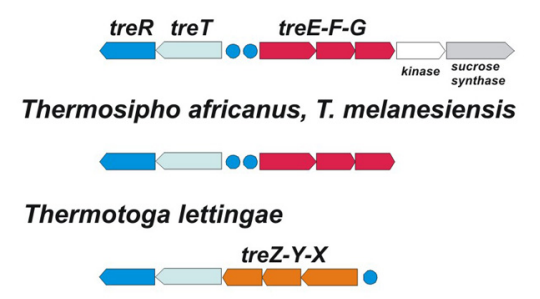

Kosmotoga olearia

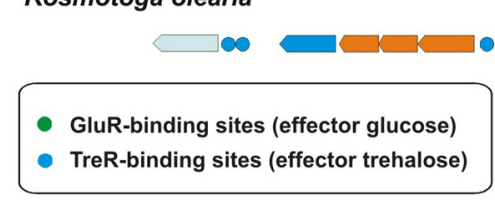

FIGURE 4 | Genomic context and conservation of trehalose and glucose utilization genes and regulons in Thermotogales.

yet uncharacterized sugar catabolic pathways (UctR/TM0326, UgtR/TM1228, and UgpR/TM1856).

\section{EXPERIMENTAL VALIDATION OF THE INFERRED REGULONS IN T. MARITIMA}

We further assessed the reconstructed sugar catabolic regulons by performing genome-wide transcriptional profiling of T. maritima cells grown on various mono- and disaccharides. First, the ability of T. maritima to grow on 17 different carbon sources was tested in minimal media with a $10-\mathrm{mM}$ concentration of the particular carbohydrate in 500-ml bottles under anoxic conditions at $80^{\circ} \mathrm{C}$. Growth phenotype testing revealed that T. maritima is able to grow on the monosaccharides rhamnose, galactose, glucose, fructose, arabinose, xylose, ribose, and mannose and the disaccharides trehalose, cellobiose, and maltose as a sole carbon and energy source (Figure 5). In contrast, $\mathrm{N}$-acetylglucosamine, gluconate, glucuronate, galacturonate, mannitol, sorbitol, and inositol did not support the growth of T. maritima and thus were not used for further transcriptional analysis. The obtained phenotypes are in agreement with the previous studies that have demonstrated growth of T. maritima on simple sugars such as glucose, mannose, ribose, arabinose, xylose, and rhamnose (Conners et al., 2005), as well as on the disaccharides maltose and lactose (Nguyen et al., 2004).

Using high-density oligonucleotide tiling arrays, we analyzed changes in transcription levels of $T$. maritima cells grown on different carbon sources (see Methods). The obtained sugarspecific transcriptomes were compared with ribose and other

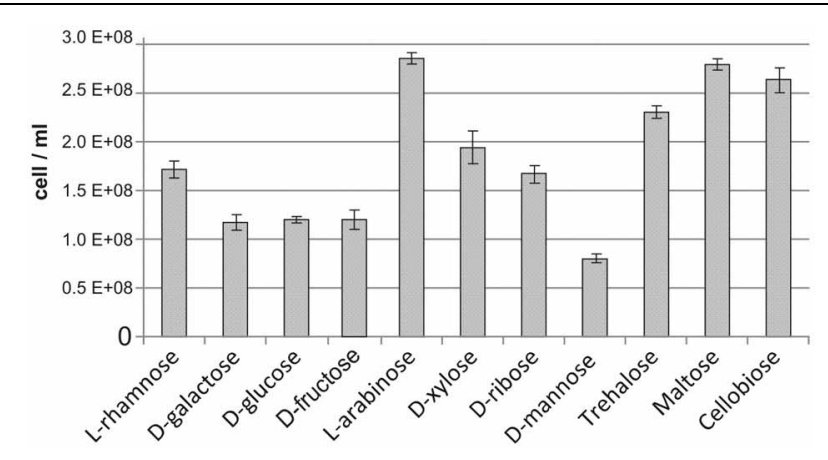

FIGURE 5 | Maximum cell concentration for $\boldsymbol{T}$. maritima grown on various carbon sources. Cell density was determined by optical density measured at $600 \mathrm{~nm}$ (OD600) after $24 \mathrm{~h}$ of cultivation. These values were converted to cell counts using the following equation [cells $/ \mathrm{mL}]=$ OD600/3.58 $\times 10^{-10}$, which was determined from measures of OD600 taken at numerous time points in the growth curve and correlated with flow cytometric cell counts.

sugars to calculate the differential expression of all genes from regulons reconstructed in T. maritima (Table S3 in Supplementary Material). The obtained gene induction patterns for xylose, cellobiose, trehalose, glucose, arabinose, ribose, rhamnose, and galactose correlate with the reconstructed regulons and metabolic effectors for the XylR, BglR, CelR, TreR, GluR, AraR, RbsR, RhaR, and GalR regulators. In addition, the UxaR regulon involving the pectin and galacturonate genes was experimentally validated in $T$. maritima by both the transcriptomic approach and the in vitro DNA-binding assays with the recombinant UxaR protein. The genes/operons from these regulons are presented in Figure 2 and discussed below with respect to their differential expression and physiological function in T. maritima.

\section{XylR regulon}

Transcription levels of all 27 genes from the seven operons regulated by the xylose-responsive transcription factor XylR were significantly elevated in T. maritima grown on xylose compared to cells grown on ribose. The highest fold changes of gene expression ( $\sim 10$ - to 20 -fold) were observed for two xylan utilization gene clusters, $x y n B<>x l o E F G K L-x y l 3-a x e A$ and $x y n A$ cen $C<>x t p F G K L E-a g u E$. The xylose catabolic gene cluster $x y l R$ adhB-xylFUEKB demonstrated modest upregulation on xylose ( $\sim 2$ - to 6-fold), whereas the xylose isomerase xylA had approximately 10 -fold higher transcript levels. The xylan oligosaccharide utilization operon $x t p N-b g a L$ was induced approximately 6-fold. In vitro, the XylR regulator responded to $0.02 \mathrm{mM}$ of xylose and also to $>0.2 \mathrm{mM}$ of glucose (Kazanov et al., 2013). However, current in vivo experiments have not identified any significant induction of the XylR-regulated genes on glucose, suggesting that xylose, rather than glucose, is a physiological inducer of the XylR regulon in T. maritima.

\section{BglR and CelR regulons}

Genes from the BglR-controlled bglREFGKL-TM0026-bglB-lamA operon were 4- to 6-fold upregulated during growth on cellobiose ( $\beta$-glucosyl-1,4-glucose) and 3- to 11-fold induced by 
glucose. The obtained gene expression profiles for the BglR regulon are corroborated by the previous in vitro DNA-binding assays with the BglR repressor that was affected by both cellobiose and glucose, although the glucose disaccharide was a more effective inducer (Kazanov et al., 2013). All 13 genes that belong to five operons from the predicted CelR regulon were highly upregulated during growth on cellobiose but remained uninduced during growth on glucose. The $c b p A$ and celQ genes showed the highest induction by cellobiose (more than 10-fold), whereas the responses of the celEFGKLR, cel12AB, and TM031213 operons to cellobiose were more moderate (5- to 9-fold). In contrast, the putative xyloglucan oligosaccharide utilization gene cluster $g l o R<>$ gloEFGKL-cel74-fucAI, which was predicted as a part of the CelR regulon, was uninduced on cellobiose or other sugars tested. The latter fact can be explained by transcriptional repression of the xlo gene locus by another LacI-family predicted regulator, GloR, that has a yet-unknown sugar effector. Based on these results, we concluded that cellobiose is a physiological effector that induces the CelR regulon in T. maritima.

\section{TreR and GluR regulons}

The TreR-regulated treTR operon encoding a putative trehalose utilization enzyme and the trehalose-responsive repressor was highly upregulated in T. maritima cells grown on trehalose (Figure 6A). Although the DNA microarray hybridization confirmed the in vivo induction of treTR by trehalose, similar analysis of the treEFG and gluEFK genes was not possible due to the absence of the respective oligonucleotide probes. The T. maritima MSB8 isolate that was originally sequenced in 1999 [TIGR genomovar, (Nelson et al., 1999)] and that was used for microarray gene probe design lacks the 8870-bp DNA region between the genes TM1846 (cbpA) and TM1847 (gluR). This DNA region is present in two other T. maritima MSB8 isolates that have recently been resequenced [genomovars DSM 3109 (Boucher and Noll, 2011) and ATCC (Latif et al., 2013)]. The new region includes seven genes including $b g l A$, a member of the CelR-regulated $c b p A$ $b g l A$ operon, and the treEFG and $g l u E F K$ genes that are regulated by TreR and GluR (Kazanov et al., 2013) (Figure 6C). To validate sugar-specific induction of these two operons in vivo, we performed real-time reverse transcription PCR (RT-PCR) with probes designed for $t r e E, g l u E$, $g l u R$, and $b g l A$ (used as a negative control) (Figure 6B). All three genes tested had demonstrated elevated expression levels in T. maritima cells grown on either glucose or trehalose compared to the cells grown on ribose. The highest fold changes in gene expression were observed for treE and $g l u E$ in the glucose-grown cells, whereas in the trehalosegrown cells only the treE gene was highly upregulated. These results confirm the dual control of the trehalose transporter treEFG by both TreR and GluR regulators in response to trehalose and glucose, respectively. Also, the RT-PCR results confirm that the glucose transporter gluEFGR operon is induced by glucose using the glucose-responsive repressor GluR.

\section{AraR regulon}

All genes from the predicted AraR regulon that are organized in three transcriptional units were upregulated in T. martima cells grown on arabinose compared to cells grown on ribose. The

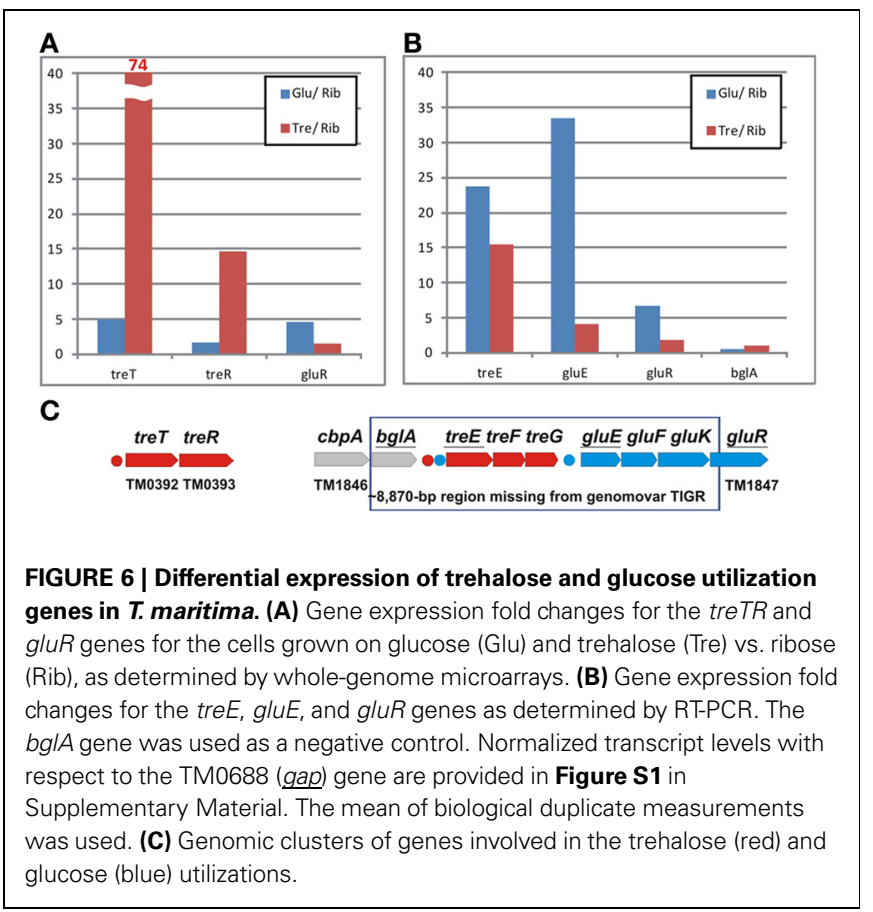

TM0280-abfA and araA genes demonstrated the highest induction levels (61- to 161-fold), the arabinoside transporter genes araEFG were modestly induced (9- to 16-fold), whereas expression of the arabinose catabolic genes araMDBW was increased only 2- to 6-fold. Large differences in the induction levels between the TM0280-abfA and araMDBW genes can be attributed to the putative transcriptional terminator upstream of araM (Latif et al., 2013). These results suggest that arabinose is a physiological inducer for the AraR regulon. This hypothesis is supported by the fact that AraR orthologs in Clostridium acetobutylicum and B. subtilis function as arabinose-responsive repressors (Zhang et al., 2012).

\section{RbsR regulon}

Transcription of all genes from the ribose utilization operon was highly upregulated in T. maritima cells grown on ribose compared to cells grown on glucose (14- to 92-fold). Unexpectedly, the ribose operon genes were almost equally highly induced in cells grown on xylose. The ribose operon is predicted to be controlled by the LacI-family regulator RbsR, whose molecular effector is not yet experimentally determined. The unusual induction patterns for ribose utilization genes may be explained by the dual specificity of the RbsR repressor to ribose and xylose. Alternatively, since the xylose utilization feeds the pentose-phosphate pathway, it is possible that ribose 5-phosphate, an intermediate of this pathway, serves as a physiological effector of the RbsR regulon in $T$. maritima.

\section{RhaR regulon}

The rhamnose oligosaccharide utilization gene cluster in T. maritima includes 14 genes organized in three transcriptional units that are predicted to be regulated by RhaR. Transcription of all three RhaR-regulated operons was highly elevated in T. maritima cells grown on rhamnose compared to cells grown on 
ribose. The rhamnose catabolic genes from the rhaMADBC operon were induced between 27- and 37-fold, the rhaR-agu4C operon showed $\sim 12$-fold induction, whereas the rtpEFGKL-gusBTM1061 operon genes demonstrated the gradual decrease in their induction level from 60 -fold for the first gene to $\sim 5$-fold for the latter gene. These expression results suggest that the RhaR regulator in T. maritima responds to the rhamnose monosaccharide.

\section{GalR regulon}

The predicted galactoside utilization regulon GalR in T. maritima includes 15 genes organized into two operons, both of which were upregulated during growth on galactose. Genes encoding putative transporters for galactose oligosaccharide and galactosides ( $g a n E F G$ and $l$ tpEFGKL), as well as two $\beta$-galactosidases (lacA and $\operatorname{gan} A)$, demonstrated the highest levels of induction on galactose compared to ribose ( 8 - to 20 -fold). The galactose catabolic genes ( $g a l T$ and $g a l K)$ and the putative galactose repressor gene galR were modestly induced by galactose $(\sim 4$-fold). These results are in agreement with the previous microarray expression profiling of T. maritima that have demonstrated induction of all abovementioned GalR regulon genes in cells grown on lactose but not on maltose (Nguyen et al., 2004).

\section{UxaR regulon}

The GntR-type transcriptional regulator UxaR was predicted to bind a conserved DNA motif in regulatory regions of four pectin and galacturonate utilization operons in T. maritima (Figures 7A,B). The UxaR regulon includes the extracytoplasmic pectate lyase PelA, the digalacturonate-specific transporter AguEFG, the cytoplasmic polygalacturonase PelB, and the extensive set of galacturonate catabolic enzymes (Rodionova et al., 2012a). We performed experimental validation of the predicted UxaR regulon by both in vitro and in vivo approaches. To assess specific binding of the purified recombinant UxaR protein to the predicted UxaR operators in T. maritima we used a fluorescence polarization assay (Figure 7C). The results show that UxaR specifically binds to the synthetic 27-nt DNA fragments containing UxaR binding sites. All tested DNA fragments demonstrated the concentration-dependent increase of fluorescence polarization, confirming specific interaction between the regulator and DNA fragments. The apparent $K_{d}$ values for the UxaR protein interacting with the tested DNA fragments were in the range of $40-80 \mathrm{nM}$. We also tested the influence of potential sugar effectors on protein-DNA interaction, however, the addition of digalacturonate, galacturonate, glucuronate, or hexuronate

A

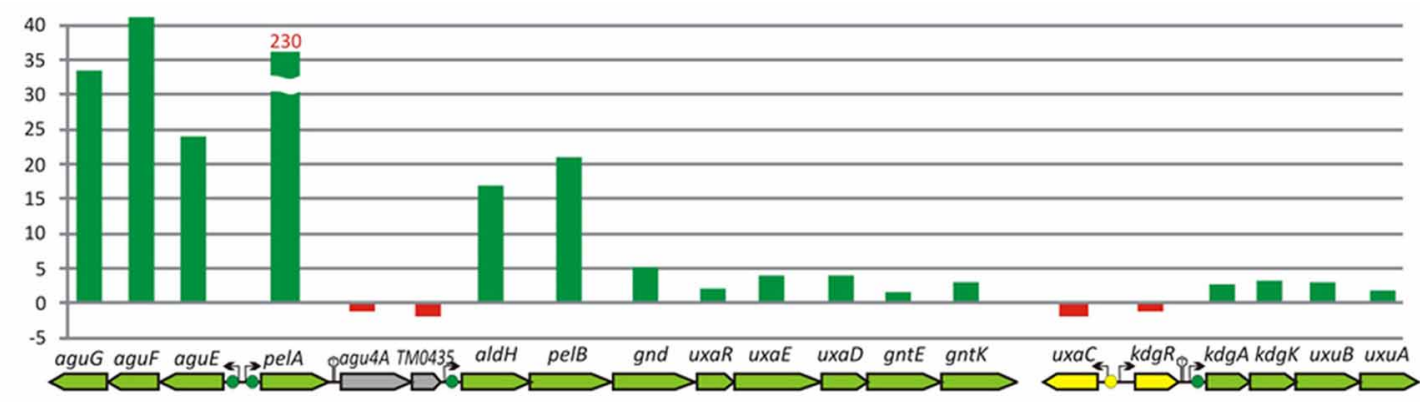

B

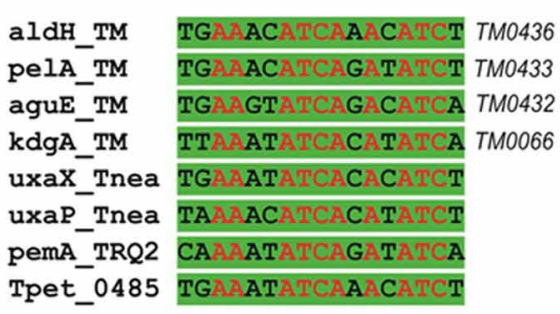

C

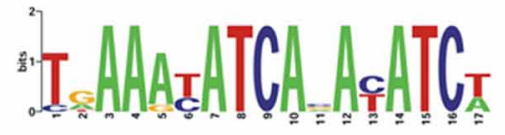

D

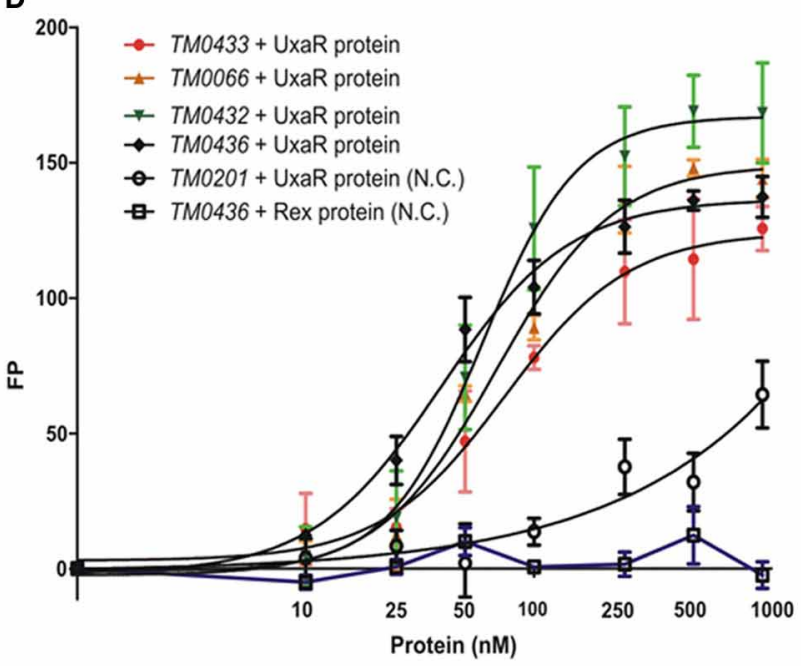

FIGURE 7 | Experimental validation of UxaR regulon in T. maritima. (A) Non-redundant set of UxaR binding sites identified in T. maritima (TM), $T$. neapolitana (Tnea), Thermotoga sp. RQ-2 (TRQ2), and T. petrophila (Tpet). (B) Sequence logo for UxaR binding sites. (C) Fluorescence polarization binding assay of UxaR with target DNA operators in T. maritima. Increasing protein concentrations were mixed with 27-bp fluorescence-labeled DNA fragments of gene regions containing UxaR-binding sites. As a negative control (N.C.), the T. maritima regulator Rex (TM0169) and DNA fragment of the TM0201 gene containing the Rex-binding site were used. (D) Gene expression fold change for T. maritima grown on pectin vs. ribose. 
catabolic pathway intermediates had no effect on the complex formation (data not shown).

Galacturonate does not support the growth of T. maritima when used as a single carbon source (data not shown). Thus, we assessed gene expression in cells grown on pectin, a galacturonatecontaining polysaccharide, which can support the growth of $T$. maritima (Kluskens et al., 2003). The expression of $\sim 50$ sugar catabolic genes was significantly ( $>2$-fold) upregulated in cells grown on pectin compared to cells grown on ribose (Table S3 in Supplementary Material). In particular, the expression of 6 genes from the UxaR regulon (aguEFG, pelA, and aldH-pelB) showed more than 17-fold upregulation (Figure 7D). The extracellular pectate lyase gene pelA was expressed 230-fold higher on pectin when compared to ribose. Most of the remaining UxaR-regulated genes from the aldH-pelB-gnd-uxaRED-gntEK and $k d g A K-u x u B A$ operons showed a 2 - to 5 -fold upregulation on pectin. The agu4ATM0435 genes located immediately downstream of pelA did not show any upregulation on pectin, suggesting that they are not co-transcribed with pelA. Accordingly, we found a putative rhoindependent transcriptional terminator downstream of pelA. In addition to the induction of pectin/galacturonate catabolic genes, three gene clusters encoding the beta-glucoside (TM0024-32), arabinose (TM0276-284), and galactoside (TM1190-1204) utilization pathways were significantly upregulated in T. maritima cells grown on pectin. These genes clusters do not belong to the UxaR regulon but were predicted to be regulated by BglR, AraR, and GalR, respectively. Induction of these three regulons by pectin may be explained by the presence of glucose, arabinose, and galactose residues in this polysaccharide.

\section{DISCUSSION}

Transcriptional regulation is a highly variable component of carbohydrate utilization networks. Although our current knowledge of sugar catabolic regulons in model bacteria such as Escherichia coli and Bacillus subtilis is nearly comprehensive, the projection of this knowledge to the sequenced genomes of bacteria from distant taxonomic groups is still a challenge. The major difficulties for bioinformatics-based propagation of TF regulons include duplications and losses of TFs and their binding sites, rapid diversification of specificities of TFs toward DNA sites and sugar effectors, non-orthologous replacements of entire regulatory systems, and frequent horizontal transfers. Comparative genomic analysis of specific sugar utilization pathways has led to substantial progress in the identification and reconstruction of their cognate TF regulons in diverse bacterial lineages (Yang et al., 2006; Rodionov et al., 2010; Leyn et al., 2012). In this study, we used comparative genomics to reconstruct novel TF regulons for sugar catabolism in hyperthermophilic bacteria from the deepbranching lineage Thermotogales (Figure 1). In T. maritima, a model bacterium in this lineage, the identified sugar catabolic regulatory network includes $18 \mathrm{TFs}, 40 \mathrm{TF}$ binding sites, and 163 target genes comprising 15 known and three hypothetical metabolic pathways (Table 1; Figures 2, 3). All studied sugarresponsive TFs in T. maritima are encoded within their cognate regulated gene loci, and thus are subject to autoregulation.

All sugar utilization regulons reconstructed in Thermotogales are controlled by TFs that are homologous to known sugar-related regulators from six families: DeoR, GntR, IclR, LacI, ROK, and RpiR. For most of these TFs, phylogenetic and genome context analysis of distant homologs did not reveal their potential functional orthologs outside the Thermotogales lineage. These genomic observations suggest that the analyzed sugar metabolic regulators have evolved and functionally specialized after the separation of the Thermotogales. Our previous phylogenetic analysis suggests that the expansion of the ROK family represented by seven paralogs in T. maritima was likely due to massive duplications and subsequent functional diversification of regulators during the evolution of Thermotogales (Kazanov et al., 2013). The DeoR-family regulator FruR controlling the fructose utilization operon in two closely related Thermotoga spp. represents an exceptional case of relatively recent horizontal gene transfer from thermophilic Clostridia. All genes from the fructose utilization operon in Thermotoga spp., including fruR, are highly similar to the orthologous genes in Caldicellulosiruptor spp. (70-80\% identity). We propose that the entire fructose utilization operon was laterally transferred between these two lineages that likely share the same ecological niche.

The observed variations in the distribution of sugar utilization regulons among the Thermotogales are also remarkable (Figure 1). Only six regulons are present in at least two different genera (CelR, ChiR, GalR, GloR, RbsR, and TreR), whereas the remaining regulons are present only in the Thermotoga genus. Moreover, most of the latter regulons are restricted to the group of five closely related Thermotoga spp, and only three regulons have orthologs in a more distant genome of T. lettingae. Conservation of all Thermotoga-specific regulons except FruR suggests their likely emergence in the common ancestor of this genus. We observed several cases of species-specific regulon loss in which an entire regulon (including all operons from a regulated pathway) is missing only in a single Thermotoga spp., including the inositol and ribose utilization regulons in the RQ-2 strain and the glucose utilization regulon GluR in T. petrophila. In another case, the RhaR regulator was lost in a single strain of Thermotoga sp. RQ-2, however, the rhamnose utilization operons are still retained in the genome. Many other cases of the absence of orthologous regulators for sugar catabolic pathways in the genomes of more distant Thermotogales can be explained by their control by non-orthologous TFs that have yet to be characterized (Figure 1).

Overall, the reconstructed carbohydrate utilization regulatory network in T. maritima contains 18 local TF regulons (FruR is absent from T. maritima), each controlling between one and seven operons. In contrast to other model bacteria (e.g., E. coli and B. subtilis) that employ various transcriptional mechanisms for global catabolite repression of sugar metabolism, our genomicbased analysis did not reveal any global regulons for sugar utilization genes in Thermotoga. This observation correlates with the genome-wide transciptome data for T. maritima obtained in this and previous studies (Chhabra et al., 2003; Frock et al., 2012). With the exception of a fructose-specific system in two Thermotoga strains, the Thermotogales genomes lack orthologs of sugar phosphotranferase (PTS) systems that are essential components of global carbon catabolic repression mechanisms in both Gram-negative and Gram-positive bacteria (Deutscher, 2008). 
Thus, bacteria from the Thermotogae phylum appear to use different regulatory strategies for sugar utilization.

Another remarkable feature of sugar catabolic networks in Thermotoga is the existence of multiple interconnections between distinct sugar regulons. Two xylan catabolic gene loci, xtp and $x$ lo, were found to be controlled by the xylose-responsive regulator XylR and the predicted glucuronate utilization regulator KdgR in T. maritima (Figure 2) and in other Thermotoga genomes (Table S2 in Supplementary Material). The first gene locus encodes a secreted endoxylanase, a putative xylan oligosaccharide $\mathrm{ABC}$ transporter, and a cytoplasmic glucuronidase, which might be involved in the cleavage of glucuronate residues from a xylan-derived oligosaccharide. The second gene locus encodes another secreted endoxylanase, a xylose oligosaccharide ABC transporter, and a cytoplasmic xylosidase and is presumably involved in the utilization of xylose-containing oligosaccharides. From transcriptomic data in T. maritima, both XylR/KdgRregulated gene loci are upregulated during growth on xylose (Tables S3 in Supplementary Material) and xylan (Conners et al., 2005). Another gene locus, glo, encoding hypothetical glucose oligosaccharide utilization genes, is predicted to be controlled by both the locally encoded regulator GloR and the distal regulator CelR, which also controls five other operons involved in cellobiose and glucan utilization (Figure 2). Lastly, the trehalose transporter treEFG in five Thermotoga spp. is controlled by the trehalose- and glucose-responsive regulators TreR and GluR, respectively (Figure 4). We confirmed by RT-PCR that the treE gene in T. maritima is upregulated on glucose and trehalose (Figure 7). The observed partial overlaps between distinct local regulons in Thermotoga point to the existence of coordinated regulatory responses to particular types of complex polysaccharides and disaccharides.

We assessed the reconstructed sugar regulatory network in T. maritima by microarray gene expression profiling of cells grown on a variety of mono- and disaccharides and pectin as a single carbon source. The obtained gene induction patterns demonstrate overall consistency with individual regulons reconstructed by genomic analysis. In addition, the previously determined effectors of the ROK-family regulators XylR, TreR, GluR, and BglR (Kazanov et al., 2013) were found to be in good agreement with the in vivo gene expression results for xylose, trehalose, glucose, and cellobiose, respectively (Figure 2). In addition, the reconstructed regulons correlated with previous microarray expression studies in T. maritima (Chhabra et al., 2003; Nguyen et al., 2004; Conners et al., 2005). For instance, the predicted galactoside utilization regulon GalR was induced by galactose in our study and was previously shown to be upregulated on lactose, a galactose-glucose disaccharide (Nguyen et al., 2004). The predicted cellobiose and glucan utilization regulon CelR was induced by cellobiose in our study and was previously shown to be upregulated by barley and glucomannan (Conners et al., 2005).

The reconstructed regulatory network allowed us to suggest and/or refine specific functional assignments for sugarspecific $A B C$ transporters that constitute the most abundant class of uptake transporters in sugar utilization pathways of Thermotogales. In T. maritima, the previously uncharacterized transporter ChiEFG was tentatively assigned chitobiose specificity based on the chitobiose-responsive regulon ChiR (Figure 3). Similarly, the characterized GluR and TreR regulons and their respective effectors allowed us to propose glucose and trehalose specificities for the GluEFK and TreEFG transporters, respectively, and these functional assignments were recently experimentally validated (Boucher and Noll, 2011). Finally, the reconstructed regulons of other Thermotoga species allowed us to predict sugar specificities for multiple novel ABC transporters that are nonorthologous to transport systems from T. maritima (Table S1 in Supplementary Material). Interestingly, despite of the absence of FruR regulon, T. maritima demonstrates some growth on fructose as a sole carbon source (Figure 5). This observation correlates with the presence of functional fructokinase TM0296 (Rodionova et al., 2012b), however, the fructose uptake transporter remains unknown in T. maritima.

In summary, the comparative genomics-based regulon and pathway reconstruction combined with some experimental data allowed us to identify the integrated metabolic and regulatory network of sugar utilization in T. maritima and revealed its substantial diversity between different Thermotogales species. Our results revealed a high level of consistency between the in silico-predicted TF regulons, the in vitro-determined TF effector specificities, and the in vivo-measured gene expression changes. The described integrative genomics-based approach for regulon analysis may be applied to other yet uncharacterized taxonomic groups of microorganisms for which multiple closely related genomes are available.

\section{MATERIALS AND METHODS} FUNCTIONAL GENE ANNOTATION AND METABOLIC RECONSTRUCTION

To map carbohydrate utilization genes in the T. maritima genome, we used the bioinformatic workflow that was previously applied for the analysis of Shewanella genomes (Rodionov et al., 2010). The workflow is based on a collection of manually curated subsystems in the SEED genomic database capturing a substantial fraction of known sugar utilization pathways projected across microbial genomes (Overbeek et al., 2005). Using a compilation of $\sim 500$ groups of isofunctional homologs from various sugar utilization subsystems in SEED, we performed homology-based scanning of the T. maritima genome. Based on genome context analysis of the identified gene candidates, we finally collected $\sim 240$ genes potentially involved in carbohydrate utilization in T. maritima. We also collected experimental knowledge and literature references for $\sim 130$ proteins from the collection including 62 sugar catabolic enzymes, 12 binding components of $\mathrm{ABC}$ transporters, and 6 regulators with established cognate metabolites (substrates, ligands, or effectors). For the remaining $\sim 50$ T. maritima proteins, the previously reported gene expression profiles revealed their upregulation during the growth on specific poly- or monosaccharides used as a single carbon source (Nguyen et al., 2004; Conners et al., 2005; Frock et al., 2012). The obtained functional gene annotations are captured in the SEED subsystem available online at http://pubseed.theseed.org/SubsysEditor.cgi?page=Show Subsystemandsubsystem=Sugarutilizationin Thermotogales and summarized in Table S1 in Supplementary Material. 


\section{GENOMIC RECONSTRUCTION OF REGULONS}

Putative TF regulons were reconstructed using the established comparative genomic approach [reviewed in (Rodionov, 2007)] implemented in the RegPredict Web server (http://regpredict.lbl. gov) (Novichkov et al., 2010b). We started from the genomic identification of reference sets of genomes that encodes orthologs of each TF and their genome context analysis. Initial training sets of potentially co-regulated genes were collected based on the gene neighborhood analysis of TF orthologs in the MicrobesOnLine database (http://microbesonline.org/) (Dehal et al., 2010). For de novo identification of a candidate TF-binding motif in the training set of potential upstream regions, we used the "Discover Profile" procedure implemented in RegPredict. Each identified DNA motif was used to scan the Thermotogales genomes to predict novel regulon members. Scores of candidate sites were calculated as the sum of positional nucleotide weights. The score threshold was defined as the lowest score observed in the training set. The conserved regulatory interactions with high-scored binding sites that involve target genes involved in carbohydrate metabolism were included in the reconstructed regulons. Candidate sites associated with new members of regulons were added to the training set, and the respective group profile was rebuilt to improve search accuracy. Sequence logos for the derived group-specific DNA binding motifs were drawn using the WebLogo package (Crooks et al., 2004). The details of all reconstructed regulons are captured and displayed in RegPrecise, a specialized database of bacterial regulons (http://regprecise.lbl. gov) (Novichkov et al., 2010a), as a part of the Thermotogales collection.

\section{DNA MICROARRAY HYBRIDIZATION}

T. maritima MSB8 (ATCC:43589) was grown in 500-ml serum bottles containing $200 \mathrm{ml}$ of minimal medium (Rinker and Kelly, 2000) with $10 \mathrm{mM}$ of a carbon source under anoxic conditions at $80^{\circ} \mathrm{C}$. Carbon sources D-glucose, D-galactose, L-rhamnose, L-arabinose, D-xylose, D-ribose, D-mannose, $\mathrm{N}$ acetyl-D-glucosamine, D-gluconate, glucuronate, galacturonate, mannitol, sorbitol, trehalose, cellobiose, maltose, and pectin were obtained from Sigma (St. Louis, MO). The cell growth was monitored spectrophometrically at $600 \mathrm{~nm}$. Once the midlogarithmic phase was reached, growth was stopped with $20 \mathrm{ml}$ of stop solution composed of 5\% Trizol and 95\% ethanol (Sigma). RNA isolation was performed using a conventional RNAeasy mini kit protocol with DNaseI treatment (Qiagen, Valencia, CA). Total RNA yields were quantified by using a NanoDrop (Thermo Fisher Scientific, Waltham, MA) at a wavelength of $260 \mathrm{~nm}$, and RNA quality was checked by measuring the samples' A260/A280 ratio (>1.8). cDNA synthesis was conducted as following: Amino-allyl cDNAs were reverse transcribed from $10 \mu \mathrm{g}$ of purified total RNA and then labeled with Cy3 monoreactive dyes (Amersham, GE HealthCare, UK). Labeled cDNA samples were fragmented to the 50-300-bp range with DNase I (EpiCentre Biotechnologies, Madison, WI) and interrogated with high-density four-plex oligonucleotide tilling arrays consisting of $4 \times 71,548$ probes of variable length spaced across the whole T. maritima genome (Roche-NimbleGen, Madison, WI). Hybridization, washing, and scanning were performed according to the manufacturer's instructions. Two biological replicates were utilized for each growth condition. Probe level data were normalized using the Robust Multiarray Analysis algorithm without background correction as implemented in NimbleScan ${ }^{\mathrm{TM}}$ 2.4 software (Roche-NimbleGen). Transcriptome data have been deposited in NCBI's Gene Expression Omnibus and are accessible through GEO Series accession number GSE47615 (http://www. ncbi.nlm.nih.gov/geo/query/acc.cgi?acc=GSE47615).

\section{REAL TIME PCR}

Individual transcript levels were measured for four genes from the ATCC-derived strain of T. maritima MSB8: cbpA (Tmari_1862), treE (Tmari_1861), gluE (Tmari_1858), and gluR (Tmari_1855) (Genbank Accession CP004077). Genomic RNA was isolated from cells grown on minimal medium supplied with either trehalose, D-glucose, or D-ribose and collected at the same optical density using the RNeasy minikit (Qiagen, Valencia, CA). Reverse transcription was performed on $10 \mu \mathrm{g}$ of total RNA. The reverse transcription mixture $(60 \mu \mathrm{l})$ contained $10 \mu \mathrm{g}$ total RNA, $75 \mu \mathrm{g}$ random primers, $1 \times 1$ st Strand buffer, $10 \mathrm{mM}$ dithiothreitol, $0.5 \mathrm{mM}$ deoxynucleoside triphosphates, $30 \mathrm{U}$ Superase, and $1,500 \mathrm{U}$ Superscript II. The mixture was incubated in a thermocycler (Bio-Rad, Hercules, CA) at $25^{\circ} \mathrm{C}$ for $10 \mathrm{~min}, 37^{\circ} \mathrm{C}$ for $1 \mathrm{~h}$, and then $42^{\circ} \mathrm{C}$ for $1 \mathrm{~h}$. The reaction was followed by incubation at $70^{\circ} \mathrm{C}$ for $10 \mathrm{~min}$ to inactivate the superscript. The RNA was then degraded by adding $20 \mu \mathrm{l}$ of $1 \mathrm{~N} \mathrm{NaOH}$ and incubating at $65^{\circ} \mathrm{C}$ for $30 \mathrm{~min}$. After the incubation, $20 \mu \mathrm{l}$ of $1 \mathrm{~N} \mathrm{HCl}$ were added to neutralize the solution. QIAquick PCR purification kits were used to clean up the cDNA synthesis product. Following the purification, the cDNA was quantified and then directly used in quantitative PCR (qPCR) reactions. The $50 \mu \mathrm{l}$ of qPCR reaction volume contained $25 \mu$ l Sybr green tag master mix (Qiagen), $0.2 \mu \mathrm{M}$ forward primer, $0.2 \mu \mathrm{M}$ reverse primer, and $\mathrm{cDNA}$ as a template. Each $\mathrm{qPCR}$ reaction was run in triplicate in the Bio-Rad thermocycler (Bio-Rad, Hercules, CA) with the following settings: $95^{\circ} \mathrm{C}$ for $15 \mathrm{~min}, 94^{\circ} \mathrm{C}$ for $15 \mathrm{~s}, 52^{\circ} \mathrm{C}$ for $30 \mathrm{~s}$, and $72^{\circ} \mathrm{C}$ for $30 \mathrm{~s}$; the denaturation, annealing, and extension steps were repeated for 40 cycles. In order to determine the binding affinity of each primer set, a standard curve was calculated for each primer and reaction efficiency obtained from it. Using the standard curve, the relative cDNA quantity was obtained for each gene by normalizing it to the quantity of gap (TM0688) cDNA in the same sample. The gap gene was used as an expression control as it demonstrated no differential expression across all gene expression arrays in the present study. Normalized transcript levels are provided in Figure S1 in Supplementary Material.

\section{GENE CLONING AND PROTEIN PURIFICATION}

The E. coli BL21 strains containing pMH2T7-derived plasmid that harbor the T. maritima TM0439 (uxaR) gene with the arabinose promoter and the $\mathrm{His}_{6}$ tag were a kind gift from Dr. S. Lesley at the Joint Center for Structural Genomics (La Jolla, CA). Recombinant UxaR protein containing an N-terminal $\mathrm{His}_{6}$ tag was overexpressed in E. coli in $50 \mathrm{ml}$ volume culture and purified using $\mathrm{Ni}^{2+}$ chelating chromatography, as previously described (Yang et al., 2008). Briefly, cells were grown in TB medium to OD600 $=1.4$ 
at $37^{\circ} \mathrm{C}$ induced by $0.15 \% \mathrm{~L}$-arabinose and harvested after $3 \mathrm{~h}$ shaking at $37^{\circ} \mathrm{C}$. Harvested cells were re-suspended in $20 \mathrm{mM}$ HEPES buffer pH 7 containing $100 \mathrm{mM} \mathrm{NaCl,} \mathrm{0.03 \%} \mathrm{Brij} \mathrm{35,}$ and $2 \mathrm{mM} \beta$-mercaptoethanol supplemented with $2 \mathrm{mM}$ PMSF and a protease inhibitor cocktail (Sigma-Aldrich). Lysozyme was added to $1 \mathrm{mg} / \mathrm{ml}$, and the cells were lyzed by freezing-thawing followed by sonication. After centrifugation at $18,000 \mathrm{rpm}$, the Tris- $\mathrm{HCl}$ buffer ( $\mathrm{pH} 8)$ was added to the supernatant $(50 \mathrm{mM}$, final concentration), and it was loaded onto an Ni-NTA acid agarose minicolumn $(0.3 \mathrm{~mL})$. After washing with the starting buffer containing $1 \mathrm{M} \mathrm{NaCl}$ and $0.3 \%$ Brij-35, bound proteins were eluted with $0.3 \mathrm{~mL}$ of the starting buffer containing $250 \mathrm{mM}$ imidazole and then followed by buffer exchange. Finally, protein was suspended in $20 \mathrm{mM}$ HEPES pH7.0 containing $1 \mathrm{mM}$ DTT and $0.5 \mathrm{mM}$ EDTA. Protein size, expression level, distribution between soluble and insoluble forms, and extent of purification were monitored by SDS PAGE. Protein concentration was determined by the Quick Start Bradford Protein Assay kit from Bio-Rad.

\section{DNA BINDING ASSAYS}

The interaction of the purified recombinant UxaR protein with their cognate DNA-binding sites in T. maritima was assessed using the fluorescence polarization DNA-binding assay. The DNA oligos containing the predicted binding sites were synthesized by Integrated DNA Technologies. One strand of oligo was $3^{\prime}$ labeled by a fluorescence label, 6-carboxyfluorescin), whereas the complementary oligo was unlabeled. The double-stranded labeled DNA fragments were obtained by annealing the labeled oligonucleotides with unlabeled complementary oligonucleotides at a 1:10 ratio. The labeled $30-b p$ DNA fragments $(1 \mathrm{nM})$ were

\section{REFERENCES}

Balk, M., Weijma, J., and Stams, A. J. (2002). Thermotoga lettingae sp. nov., a novel thermophilic, methanol-degrading bacterium isolated from a thermophilic anaerobic reactor. Int. J. Syst. Evol. Microbiol. 52, 1361-1368. doi: 10.1099/ijs.0.02165-0

Boucher, N., and Noll, K.M. (2011). Ligands of thermophilic $\mathrm{ABC}$ transporters encoded in a newly sequenced genomic region of Thermotoga maritima MSB8 screened by differential scanning fluorimetry. Appl. Environ. Microbiol. 77, 6395-6399. doi: 10.1128/AEM.05418-11

Chhabra, S. R., Shockley, K. R., Conners, S. B., Scott, K.L., Wolfinger, R. D., and Kelly, R. M. (2003). Carbohydrate-induced differential gene expression patterns in the hyperthermophilic bacterium Thermotoga maritima. J. Biol. Chem. 278, 7540-7552. doi: 10.1074/jbc.M211748200

Chhabra, S. R., Shockley, K. R., Ward, D. E., and Kelly, R. M. (2002). Regulation of endo-acting glycosyl

incubated with increasing concentrations of the purified UxaR protein $(10-500 \mathrm{nM})$ in a total volume of $100 \mu \mathrm{l}$ of the binding buffer containing $20 \mathrm{mM}$ Tris- $\mathrm{HCl}(\mathrm{pH} 7.5), 100 \mathrm{mM} \mathrm{NaCl}$, and $0.3 \mathrm{mg} / \mathrm{ml} \mathrm{BSA}$ at $37^{\circ} \mathrm{C}$ for $30 \mathrm{~min}$. Poly $(\mathrm{dI}-\mathrm{dC})$ was added to the reaction mixture as a non-specific competitor DNA at $1 \mu \mathrm{g}$ to suppress non-specific binding. The fluorescence-labeled DNA was detected with the FLA-5100 fluorescent image analyzer. A DNA fragment containing the Rex regulator binding site was used as a negative control (Ravcheev et al., 2012).

\section{ACKNOWLEDGMENTS}

We would like to thank Olga Zagnitko from the Fellowship for Interpretation of Genomes (FIG) for help with functional gene annotations and subsystem analysis in the SEED database. We are grateful to Pavel Novichkov from Lawrence Berkeley National Laboratory for help with visualization of regulons in the RegPrecise database and to Haythem Latif for deposition of microarray datasets to GEO. This work was supported by the Office of Science and Office of Biological and Environmental Research of the U.S. Department of Energy under contracts DE-FG02-08ER64686 and DE-SC0004999. Additional funding was provided by the Russian Foundation for Basic Research (12-04-33003) and by National Institute of General Medical Sciences (NIGMS) under Protein Structure Initiative (U54 GM094586-04).

\section{SUPPLEMENTARY MATERIAL}

The Supplementary Materials for this article can be found online at: http://www.frontiersin.org/Microbial_Physiology_ and_Metabolism/10.3389/fmicb.2013.00244/abstract

Dehal, P. S., Joachimiak, M. P., Price, M. N., Bates, J. T., Baumohl, J. K., Chivian, D., et al. (2010). MicrobesOnline: an integrated portal for comparative and functional genomics. Nucleic Acids Res. 38, D396-D400. doi: 10.1093/nar/gkp919

Deutscher, J. (2008). The mechanisms of carbon catabolite repression in bacteria. Curr. Opin. Microbiol. 11, 87-93. doi: 10.1016/j.mib.2008.02.007

Faria, J. P., Overbeek, R., Xia, F., Rocha, M., Rocha, I., and Henry, C. S. (2013). Genome-scale bacterial transcriptional regulatory networks: reconstruction and integrated analysis with metabolic models. Brief Bioinform. doi: 10.1093/ bib/bbs071. [Epub ahead of print].

Frock, A. D., Gray, S. R., and Kelly, R. M. (2012). Hyperthermophilic Thermotoga species differ with respect to specific carbohydrate transporters and glycoside hydrolases. Appl. Environ. Microbiol. 78, 1978-1986. doi: 10.1128/AEM.07069-11
Huber, R., Langworthy, T. A., Konig, H., Thomm, M., Woese, C. R., Sleytr, U. B., et al. (1986). Thermotoga maritima sp. nov. represents a new genus of unique extremely thermophilic eubacteria growing up to $90^{\circ} \mathrm{C}$. Arch. Microbiol. 144, 324-333. doi: 10.1007/BF00409880

Jannasch, H. W., Huber, R., Belkin, S., and Stetter, K. O. (1988). Thermotoga neapolitana sp. nov. of the extremely thermophilic eubacterial genus Thermotoga. Arch. Microbiol. 150, 103-104. doi: 10.1007/BF00409725

Kazanov, M., Li, X., Gelfand, M. Osterman, A., and Rodionov, D. (2013). Functional diversification of ROK-family transcriptional regulators of sugar catabolism in the Thermotogae phylum. Nucleic Acids Res. 41, 790-803. doi: 10.1093/nar/gks1184

Kluskens, L. D., Van Alebeek, G. J., Voragen, A. G., De Vos, W. M., and Van Der Oost, J. (2003). Molecular and biochemical characterization of the thermoactive family 1 pectate lyase from the hyperthermophilic bacterium Thermotoga maritima. 
Biochem. J. 370, 651-659. doi: 10.1042/BJ20021595

Latif, H., Lerman, J. A., Portnoy, V. A., Tarasova, Y., Nagarajan, H., Schrimpe-Rutledge, A. C., et al. (2013). The genome organization of Thermotoga maritima reflects its lifestyle. PLoS Genet. 9:e1003485. doi: 10.1371/journal.pgen.1003485

Leyn, S. A., Gao, F., Yang, C., and Rodionov, D. A. (2012). Nacetylgalactosamine utilization pathway and regulon in proteobacteria: genomic reconstruction and experimental characterization in Shewanella. J. Biol. Chem. 287, 28047-28056. doi: 10.1074/jbc.M112.382333

Leyn, S. A., Kazanov, M. D., Sernova, N. V., Ermakova, E. O., Novichkov, P. S., and Rodionov, D. A. (2013). Genomic reconstruction of transcriptional regulatory network in Bacillus subtilis. J. Bacteriol. 195, 2463-2473. doi: 10.1128/ JB.00140-13

Nelson, K. E., Clayton, R. A., Gill, S. R., Gwinn, M. L., Dodson, R. J., Haft, D. H., et al. (1999). Evidence for lateral gene transfer between Archaea and bacteria from genome sequence of Thermotoga maritima. Nature 399, 323-329. doi: 10.1038/20601

Nguyen, T. N., Ejaz, A. D., Brancieri, M. A., Mikula, A. M., Nelson, K. E., Gill, S. R., et al. (2004). Wholegenome expression profiling of Thermotoga maritima in response to growth on sugars in a chemostat. J. Bacteriol. 186, 4824-4828. doi: $\quad 10.1128 / J B \cdot 186.14 .4824-4828$. 2004

Novichkov, P. S., Laikova, O. N., Novichkova, E. S., Gelfand, M. S., Arkin, A. P., Dubchak, I., et al. (2010a). RegPrecise: a database of curated genomic inferences of transcriptional regulatory interactions in prokaryotes. Nucleic Acids Res. 38, D111-D118. doi: 10.1093/nar/gkp894

Novichkov, P. S., Rodionov, D. A., Stavrovskaya, E. D., Novichkova, E. S., Kazakov, A. E., Gelfand, M. S., et al. (2010b). RegPredict: an integrated system for regulon inference in prokaryotes by comparative genomics approach. Nucleic Acids Res. 38, W299-W307. doi: 10.1093/nar/gkq531

Overbeek, R., Begley, T., Butler, R. M., Choudhuri, J. V., Chuang, H. Y., Cohoon, M., et al. (2005). The subsystems approach to genome annotation and its use in the project to annotate 1000 genomes.
Nucleic Acids Res. 33, 5691-5702. doi: 10.1093/nar/gki866

Patel, B. K. C., Morgan, H. W., and Daniel, R. M. (1985). Fervidobacterium nodosum gen. nov. and spec. nov., a new chemoorganotrophic, caldoactive, anaerobic bacterium. Arch. Microbiol. 141, 63-69. doi: 10.1007/BF00446741

Pauly, M., and Keegstra, K. (2010). Plant cell wall polymers as precursors for biofuels. Curr. Opin. Plant Biol. 13, 305-312. doi: 10.1016/j.pbi.2009.12.009

Ravcheev, D. A., Best, A. A., Sernova, N. V., Kazanov, M. D., Novichkov, P. S., and Rodionov, D. A. (2013). Genomic reconstruction of transcriptional regulatory networks in lactic acid bacteria. $B M C$ Genomics 14:94. doi: 10.1186/14712164-14-94

Ravcheev, D. A., Li, X., Latif, H., Zengler, K., Leyn, S. A., Korostelev, Y. D., et al. (2012). Transcriptional regulation of central carbon and energy metabolism in bacteria by redox-responsive repressor Rex. J. Bacteriol. 194, 1145-1157. doi: 10.1128/JB.06412-11

Rinker, K. D., and Kelly, R. M. (2000). Effect of carbon and nitrogen sources on growth dynamics and exopolysaccharide production for the hyperthermophilic archaeon Thermococcus litoralis and bacterium Thermotoga maritima. Biotechnol. Bioeng. 69, 537-547.

Rodionov, D. A. (2007). Comparative genomic reconstruction of transcriptional regulatory networks in bacteria. Chem. Rev. 107, 3467-3497. doi: 10.1021/cr068309+

Rodionov, D. A., Gelfand, M. S., and Hugouvieux-CottePattat, N. (2004). Comparative genomics of the KdgR regulon in Erwinia chrysanthemi 3937 and other gamma-proteobacteria. Microbiology 150, 3571-3590. doi: 10.1099/mic.0.27041-0

Rodionov, D. A., Kurnasov, O. V., Stec, B., Wang, Y., Roberts, M. F., and Osterman, A. L. (2007). Genomic identification and in vitro reconstitution of a complete biosynthetic pathway for the osmolyte dimyo-inositol-phosphate. Proc. Natl. Acad. Sci. U.S.A. 104, 4279-4284. doi: 10.1073/pnas. 0609279104

Rodionov, D. A., Mironov, A. A., and Gelfand, M. S. (2001). Transcriptional regulation of pentose utilisation systems in the
Bacillus/Clostridium group of bacteria. FEMS Microbiol. Lett. 205, 305-314. doi: 10.1111/j.15746968.2001.tb10965.x

Rodionov, D. A., Novichkov, P. S., Stavrovskaya, E. D., Rodionova, I. A., Li, X., Kazanov, M. D., et al. (2011). Comparative genomic reconstruction of transcriptional networks controlling central metabolism in the Shewanella genus. $B M C$ Genomics 12(Suppl 1):S3. doi: 10.1186/1471-2164-12-S1-S3

Rodionov, D. A., Yang, C., Li, X., Rodionova, I. A., Wang, Y., Obraztsova, A. Y., et al. (2010). Genomic encyclopedia of sugar utilization pathways in the Shewanella genus. BMC Genomics 11:494. doi: 10.1186/1471-2164-11-494

Rodionova, I. A., Leyn, S. A., Burkart, M. D., Boucher, N., Noll, K. M., Osterman, A. L., et al. (2013). Novel inositol catabolic pathway in Thermotoga maritima. Environ. Microbiol. 15, 2254-2266. doi: 10.1111/1462-2920.12096

Rodionova, I. A., Scott, D. A., Grishin, N. V., Osterman, A. L., and Rodionov, D. A (2012a). Tagaturonate-fructuronate epimerase UxaE, a novel enzyme in the hexuronate catabolic network in Thermotoga maritima. Environ. Microbiol. 14, 2920-2934. doi: 10.1111/j.1462-2920.2012.02856.x

Rodionova, I. A., Yang, C., Li, X., Kurnasov, O. V., Best, A. A., Osterman, A. L., et al. (2012b). Diversity and versatility of the Thermotoga maritima sugar kinome. J. Bacteriol. 194, 5552-5563. doi: 10.1128/JB.01136-12

Swithers, K. S., Dipippo, J. L., Bruce, D. C., Detter, C., Tapia, R., Han, S., et al. (2011). Genome sequence of Thermotoga sp. strain RQ2, a hyperthermophilic bacterium isolated from a geothermally heated region of the seafloor near Ribeira Quente, the Azores. J. Bacteriol. 193, 5869-5870. doi: 10.1128/JB.05923-11

Takahata, Y., Nishijima, M., Hoaki, T., and Maruyama, T. (2000). Distribution and physiological characteristics of hyperthermophiles in the Kubiki oil reservoir in Niigata, Japan. Appl. Environ. Microbiol. 66, 73-79. doi: 10.1128/AEM.66.1.73-79.2000

Yang, C., Rodionov, D. A., Li, X., Laikova, O. N., Gelfand, M. S., Zagnitko, O. P., et al. (2006).
Comparative genomics and experimental characterization of $\mathrm{N}$-acetylglucosamine utilization pathway of Shewanella oneidensis. J. Biol. Chem. 281, 29872-29885. doi: 10.1074/jbc.M605052200

Yang, C., Rodionov, D. A., Rodionova, I. A., Li, X., and Osterman, A. L. (2008). Glycerate 2-kinase of Thermotoga maritima and genomic reconstruction of related metabolic pathways. J. Bacteriol. 190, 1773-1782. doi: 10.1128/JB.01469-07

Zhang, L., Leyn, S. A., Gu, Y., Jiang, W., Rodionov, D. A., and Yang, C. (2012). Ribulokinase and transcriptional regulation of arabinose metabolism in Clostridium acetobutylicum. J. Bacteriol. 194, 1055-1064. doi: 10.1128/JB.06241-11

Zhang, Y., Thiele, I., Weekes, D., Li, Z., Jaroszewski, L., Ginalski, K., et al. (2009). Three-dimensional structural view of the central metabolic network of Thermotoga maritima. Science 325, 1544-1549. doi: $10.1126 /$ science.1174671

Conflict of Interest Statement: The authors declare that the research was conducted in the absence of any commercial or financial relationships that could be construed as a potential conflict of interest.

Received: 13 June 2013; accepted: 31 July 2013; published online: 23 August 2013. Citation: Rodionov DA, Rodionova IA, Li X, Ravcheev DA, Tarasova Y, Portnoy VA, Zengler $K$ and Osterman AL (2013) Transcriptional regulation of the carbohydrate utilization network in Thermotoga maritima. Front. Microbiol. 4:244. doi: 10.3389/fmicb.2013.00244 This article was submitted to Microbial Physiology and Metabolism, a section of the journal Frontiers in Microbiology. Copyright (C) 2013 Rodionov, Rodionova, Li, Ravcheev, Tarasova, Portnoy, Zengler and Osterman. This is an open-access article distributed under the terms of the Creative Commons Attribution License (CC BY). The use, distribution or reproduction in other forums is permitted, provided the original author(s) or licensor are credited and that the original publication in this journal is cited, in accordance with accepted academic practice. No use, distribution or reproduction is permitted which does not comply with these terms. 